\title{
Angiotensin II promotes the progression of human gastric cancer
}

\author{
MING-MIN HUANG ${ }^{1}$, AI-BIN GUO $^{1}$, JUN-FENG SUN ${ }^{2}$, XIAO-LIN CHEN $^{1}$ and ZHEN-YU YIN ${ }^{1}$ \\ ${ }^{1}$ Department of Gerontics, Drum Tower Hospital, College of Medicine, Nanjing University, Nanjing, Jiangsu 210008; \\ ${ }^{2}$ Department of Surgical Oncology, Zhejiang University School of Medicine, Hangzhou, Zhejiang 310003, P.R. China
}

Received August 9, 2013; Accepted December 17, 2013

DOI: $10.3892 / \mathrm{mmr} .2014 .1891$

\begin{abstract}
The renin-angiotensin system (RAS) plays an important role in cardiovascular homeostasis, carcinogenesis-related angiogenesis and cell proliferation. The present study was undertaken to determine the expression of angiotensin (Ang) II, Ang II type 1 and 2 receptors (AT1R and AT2R), and the activity of the angiotensin-converting enzyme (ACE) in gastric cancer tissue. The study further examined the roles of Ang II in the growth of gastric cancer cells in nude mice and in the migration and proliferation of MKN45 human gastric cancer cells. Gastric cancer tissue samples were obtained from gastric cancer patients. The levels of Ang II, AT1R and AT2R, as well as ACE activity were increased in tissues from gastric cancer patients compared to healthy tissues. A gastric cancer model was established by intraperitoneally injecting MKN45 human gastric cancer cells in nude mice, intraperitoneally injecting Ang II and measuring the tumor size every two days. Ang II treatment caused an increase in the size and weight of the tumor mass in nude mice, whereas the AT1R antagonist losartan significantly inhibited the size and weight of the tumor. While Ang II enhanced the migratory and proliferative rate of MKN45 human gastric cancer cells, these were significantly reduced following treatment with losartan. These results indicate that RAS is activated in gastric cancer patients and Ang II promotes the progression of gastric cancer in nude mice, as well as the migration and proliferation of MKN45 human gastric cancer cells.
\end{abstract}

\section{Introduction}

Cancer is a major health issue worldwide and the second leading cause of death after cardiovascular diseases (1). Gastric cancer is one of the most common cancer types, commonly diagnosed in advanced stages where curative treatment is ineffective (2). Genetic factors (oncogenes and genes coding for inflammatory

Correspondence to: Dr Zhen-Yu Yin, Department of Gerontics, Drum Tower Hospital, College of Medicine, Nanjing University, 321 Zhongshan Road, Nanjing, Jiangsu 210008, P.R. China

E-mail: hmmglyy@163.com

Key words: gastric cancer, renin-angiotensin system, angiotensin II, proliferation, migration cytokines), infection (hepatitis virus, Helicobacter pylori and Epstein-Barr virus) and environmental factors (alcohol, salt intake and smoking) may contribute to gastric cancer (3-7).

Self-sufficiency of growth signals and metastasis are hallmark alterations in cell physiology associated with cancer (8), and involve unlimited proliferation, subsequent dissociation of tumor cells from the primary tumor and eventually, invasion of adjacent tissues. It has been shown that microRNAs $(9,10)$ and genes coding for the estrogen receptor (ER) $\alpha$ (11) and ghrelin (12) are involved in the migration and proliferation of gastric cancer cells. However, the mechanisms underlying these events remain unclear.

Therenin-angiotensin system(RAS) consists of renin, angiotensinogen, angiotensin (Ang), and the angiotensin-converting enzyme (ACE). RAS influences cardiovascular homeostasis and is associated with cardiovascular diseases such as heart failure and hypertension $(13,14)$. A major regulatory component of RAS is Ang II, a biologically active peptide that is involved in the regulation of cell proliferation, angiogenesis, inflammation, and tissue remodeling via binding to the Ang II type 1 receptor (AT1R) (15-17). An association between RAS function and gastric cancer has been shown in a number of studies (18-20). It was previously shown that ACE, AT1R and AT2R are upregulated in tumor tissues (18). Gastric mucosal ATR expression was also shown to gradually increase during the course of $H$. pylori infection; RAS activation during the progression of gastric inflammation supports its potential involvement in gastric carcinogenesis (19). Moreover, local production of Ang II in gastric cancer cells is involved in cell proliferation and survival (20). However, the relationship between Ang II and gastric cancer is not well understood.

The present study was designed to determine the roles of Ang II in the growth of gastric cancer cells in male nude mice and in the migration and proliferation of MKN45 human gastric cancer cells.

\section{Materials and methods}

Tissue specimens. Twenty gastric cancer and corresponding normal gastric mucosa tissue samples ( $>10 \mathrm{~cm}$ away from the edge of the cancer) were obtained from gastric cancer patients, snap-frozen in liquid nitrogen and stored at $-80^{\circ} \mathrm{C}$ until use. All the patients had undergone preoperative clinical staging assessment with endoscopic ultrasonography and multislice spiral computed tomography and had not received chemotherapy or radiotherapy prior to surgery. 
Animals and gastric cancer model. Male BALB/c nude mice (4-6 weeks old, 15-20 g average weight) were purchased from the Chinese Academy of Medical Sciences Laboratory Animal Center. The mice were housed in a temperature- and humidity-controlled room with a 12-h on-off light cycle and were given free access to food and water. To establish the gastric cancer mouse model, $100 \mu 1 \mathrm{MKN} 45$ gastric cancer cells in phosphate-buffered saline (PBS; $5 \times 10^{7}$ cells $/ \mathrm{ml}$ ) were intraperitoneally injected into the nude mice, as previously described (21). Normotensive sham-operated mice (PBS group) underwent a similar surgical process, except for the injection of gastric cancer cells. Tumor size was calculated as previously described (22): length $\mathrm{x}$ width $\mathrm{x}$ depth $\mathrm{x} 0.5236$.

Cell lines and cell culture. MKN45 human gastric cancer cell lines were purchased from the Cancer Institute of the Chinese Academy of Medical Sciences (Beijing, China). The cell lines were cultured in phenol red-free Dulbecco's modified Eagle's medium (DMEM) supplemented with $10 \%$ charcoal-stripped fetal bovine serum (FBS) (both from Invitrogen Life Technologies, Carlsbad, CA, USA) ), $100 \mathrm{U} / \mathrm{ml}$ penicillin and $100 \mathrm{U} / \mathrm{ml}$ streptomycin, in a humid chamber at $37^{\circ} \mathrm{C}$ under $5 \% \mathrm{CO}_{2}$. The cells were subcultured every 3-5 days to maintain logarithmic growth until a sufficient number $\left(5 \times 10^{7}\right.$ cells $\left./ \mathrm{ml}\right)$ was obtained for transfer to nude mice.

Quantification of Ang II. Gastric cancer tissues were homogenized in lysis buffer and then centrifuged (2,000 x $\mathrm{g}$ for $10 \mathrm{~min})$. The total protein in the supernatant was extracted and measured using a protein assay kit (BCA; Pierce Chemical Co., Rockford, IL, USA). The level of Ang II in the gastric cancer or healthy tissue supernatant was measured using an enzyme-linked immunosorbent assay (ELISA) kit (USCN Life Science Inc., Wuhan, China) following the manufacturer's instructions.

Measurement of ACE activity. ACE activity was measured using an ACE assay kit (Thermo Fisher Scientific Inc., Middletown, VA, USA). Briefly, $10 \mathrm{ml}$ of ACE control, calibrator or sample was added to $100 \mathrm{ml}$ of ACE reagent in a 96-well microtiter plate on ice, in triplicate. The reagents were mixed, incubated at $37^{\circ} \mathrm{C}$ and absorbance was measured at $340 \mathrm{~nm}$ at $1-\mathrm{min}$ intervals. Time plots of the average absorbance values of the control, the calibrator and the samples were used to calculate the $\Delta \mathrm{A} / \Delta \mathrm{t}$ ratios following linear regression model fitting. The ACE activity of plasma samples was determined by comparing the $\Delta \mathrm{A} / \Delta \mathrm{t}$ of each sample to the respective standard.

Western blotting. Gastric cancer tissues were lysed in modified RIPA buffer or directly in 1X SDS loading buffer. Following electrophoresis and transfer to a nitrocellulose membrane, proteins were probed with the AT1R or AT2R primary antibody (1:300; Santa Cruz Biotechnology, Inc., Santa Cruz, CA, USA), followed by incubation with the secondary antibodies (1:5,000; Immunology Consultants Laboratory, Inc., Newberg, OR, USA). The bands were visualized using the enhanced chemiluminescence (ECL) substrate (Pierce Chemical Co.) and captured on X-ray film. The total AT1R or AT2R protein level was normalized to the protein level of glyceraldehyde 3-phosphate dehydrogenase (GAPDH; Bioworld Technology Inc., St. Louis Park, MN, USA).
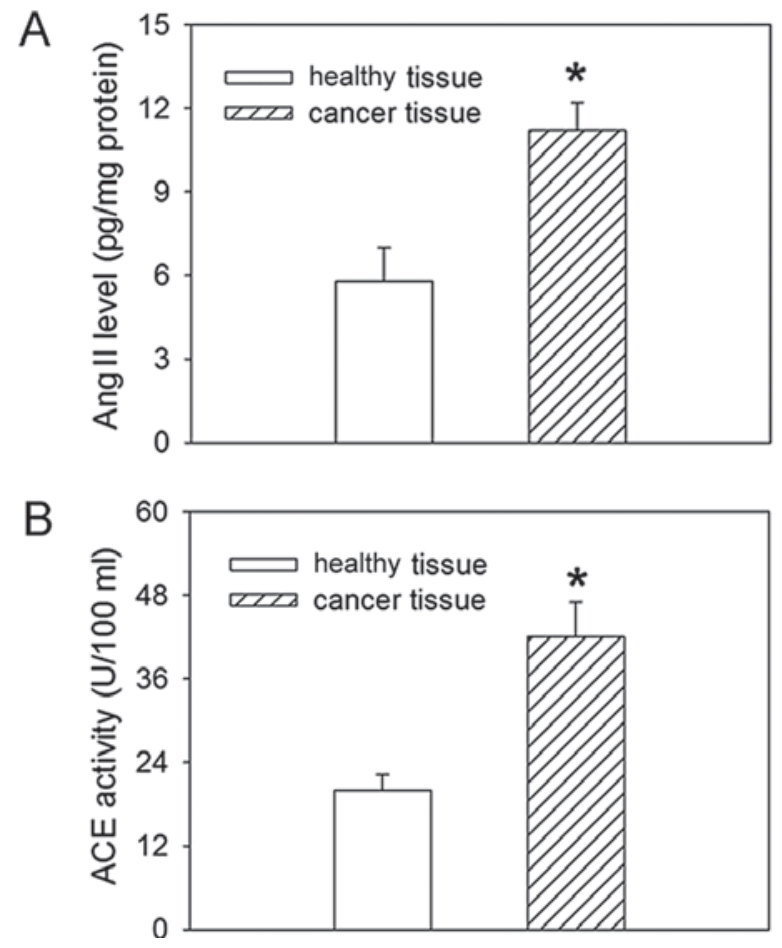

Figure 1. (A) Angiotensin II (Ang II) level and (B) angiotensin-converting enzyme (ACE) activity in tissues from gastric cancer patients and healthy controls. Values represent mean $\pm \mathrm{SE}$. ${ }^{*} \mathrm{P}<0.05$ vs. healthy gastric mucosa sample.

Cell proliferation assay. Cell proliferation was assessed by measuring bromodeoxyuridine (BrdUrd) incorporation using a BrdUrd ELISA colorimetric assay (Roche Diagnostics Corp., Indianapolis, IN, USA). Cells were initially plated at a density of $2 \times 10^{5} / 60-\mathrm{mm}$ dish. Following incubation, cells were counted using a hemocytometer and corresponding data were plotted.

Cell migration assay. Cells ( $10^{5}$ cells/well) were suspended in $0.5 \mathrm{ml}$ of $1 \%$ FBS MEM and placed in the upper compartment of a Boyden chamber (Corning Costar, Rochester, NY, USA); $0.75 \mathrm{ml}$ of $10 \%$ FBS MEM was added to the bottom compartment. Following a 48-h incubation, non-migrating cells were scraped from the membrane of the upper compartment, and cells that had migrated through the membrane were fixed and stained with the Diff-Quik 3 stain set following the manufacturer's protocol (Siemens Healthcare Diagnostics Inc., Deerfield, IL, USA). Membranes were excised and mounted on a standard microscope slide (Curtis Matheson Scientific Inc., Jessup, MD, USA). The number of cells that had migrated was determined from 5 random high-power fields (HPFs) visualized at x200 magnification.

Chemicals. Ang II and losartan (1 and $5 \mu \mathrm{mol}$, respectively) were purchased from Sigma Chemical Co. (St. Louis, MO, USA). All chemicals were dissolved in PBS and were produced on the day of the experiment $\left(37^{\circ} \mathrm{C}\right)$.

Statistical analysis. Comparisons between two observations were analyzed by Student's paired t-test, and multiple comparisons with a one- or two-way ANOVA. Multiple testing bias was assessed with the Bonferroni test. Data are expressed as 

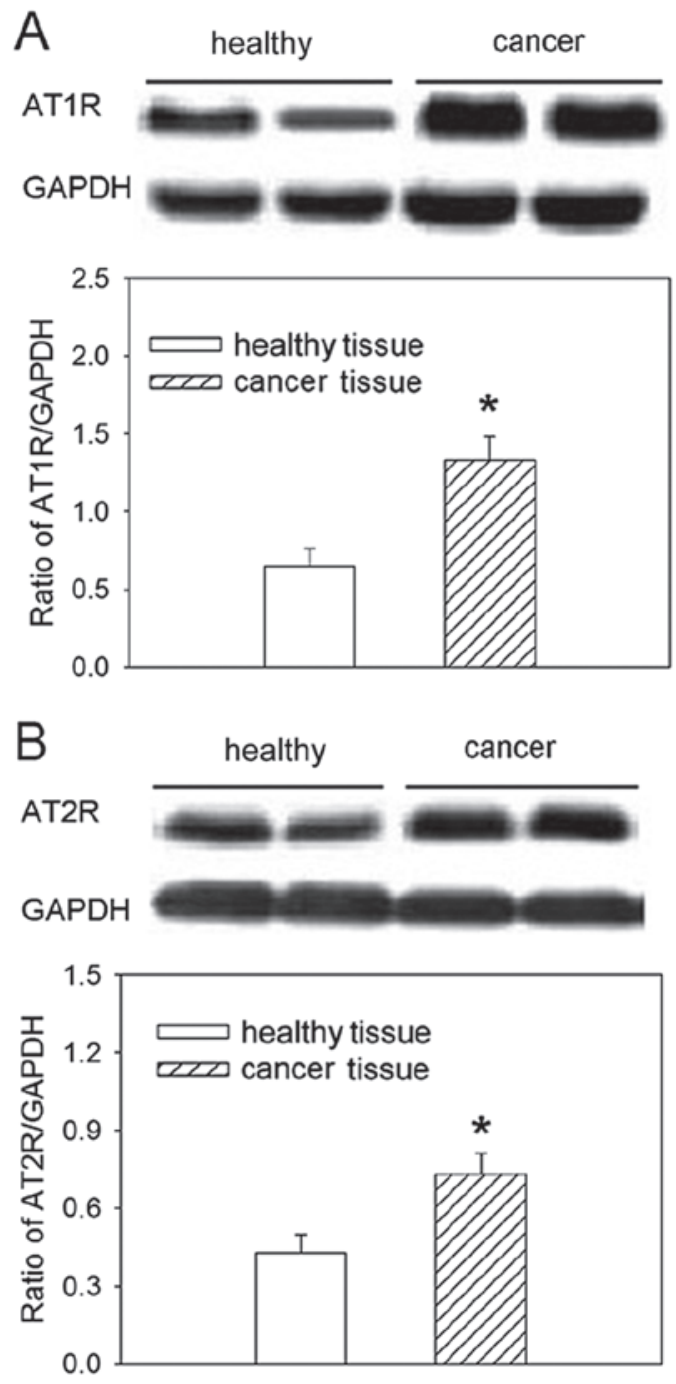

Figure 2. (A) Angiotensin II (Ang II) type 1 and (B) Ang II type 2 receptor expression in tissues from gastric cancer patients and healthy controls. Values represent mean $\pm \mathrm{SE}$. " $\mathrm{P}<0.05$ vs. healthy gastric mucosa sample.

mean \pm standard error (SE). $\mathrm{P}<0.05$ was considered to indicate statistically significant differences.

\section{Results}

Ang II level and ACE activity in tissues of gastric cancer patients. The level of Ang II in gastric cancer tissues was increased compared to that observed in healthy gastric mucosa tissues. ACE activity was also increased in gastric cancer tissues compared to healthy controls (Fig. 1).

$A T 1 R$ and AT2R expression in gastric cancer patient tissues. The relative expression of both AT1R and ATR2 proteins was increased in tissues from gastric cancer patients, compared to healthy tissues from the gastric mucosa (Fig. 2).

Effects of Ang II and losartan on tumor size and weight. Intraperitoneal injection of Ang II caused an increase in the size of tumor from the 11th day after injection (Fig. 3). The mean tumor weight was increased after 3 weeks of treatment compared to the PBS-treated control, whereas the AT1R
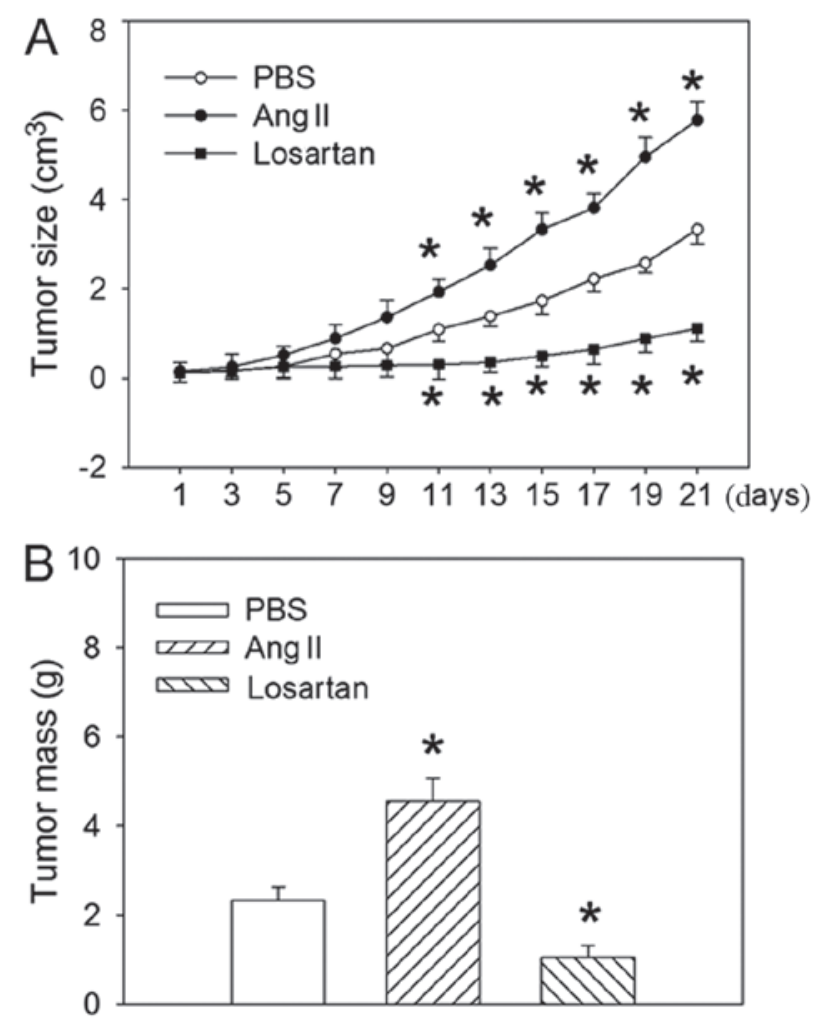

Figure 3. Effects of angiotensin II (Ang II) and the Ang II antagonist losartan on (A) tumor size and (B) mass (weight). The tumor size was measured every other day and the tumor weight was measured 3 weeks after treatment. Values represent mean $\pm \mathrm{SE}$. "P<0.05 vs. phosphate-buffered saline (PBS).

antagonist losartan significantly inhibited the size of tumor from the 11th day after injection and the mean tumor weight after 3 weeks of treatment.

Effects of Ang II and losartan on migration and proliferation. Ang II treatment increased, whereas losartan reduced the migration of MKN45 human gastric cancer cells. Treatment with Ang II also promoted proliferation as compared to PBS treatment at both 48 and $72 \mathrm{~h}$, while losartan inhibited the proliferation of MKN45 cells (Figs. 4 and 5).

\section{Discussion}

Gastric cancer is one of the most common cancer types and has been associated with genetic factors, environmental factors and infection (3-7). The RAS regulatory component Ang II is a biologically active peptide that is involved in the regulation of cell proliferation, angiogenesis, and tissue remodeling $(16,17)$. Previous studies $(18-20)$ have shown that RAS is associated with gastric cancer. It has also been shown that the ACE inhibitor and AT1R antagonist suppress gastric cancer growth $(23,24)$. Our study presents new findings on the levels of expression of Ang II, AT1R and AT2R, all found increased in tissues from gastric cancer patients. We further showed that Ang II promotes the size and weight of gastric cancer tumors in gastric cancer mice and increases the migration and proliferation of MKN45 human gastric cancer cells. Ang II thus promotes the progression of human gastric cancer. 


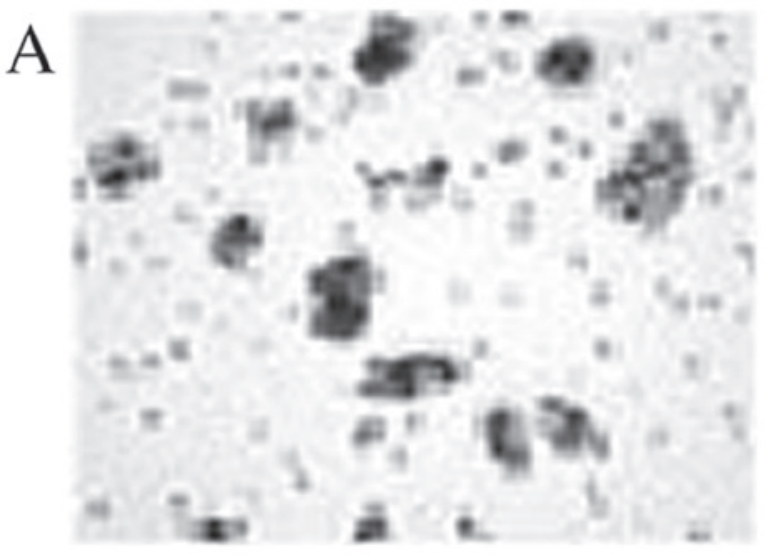

$\mathrm{B}$
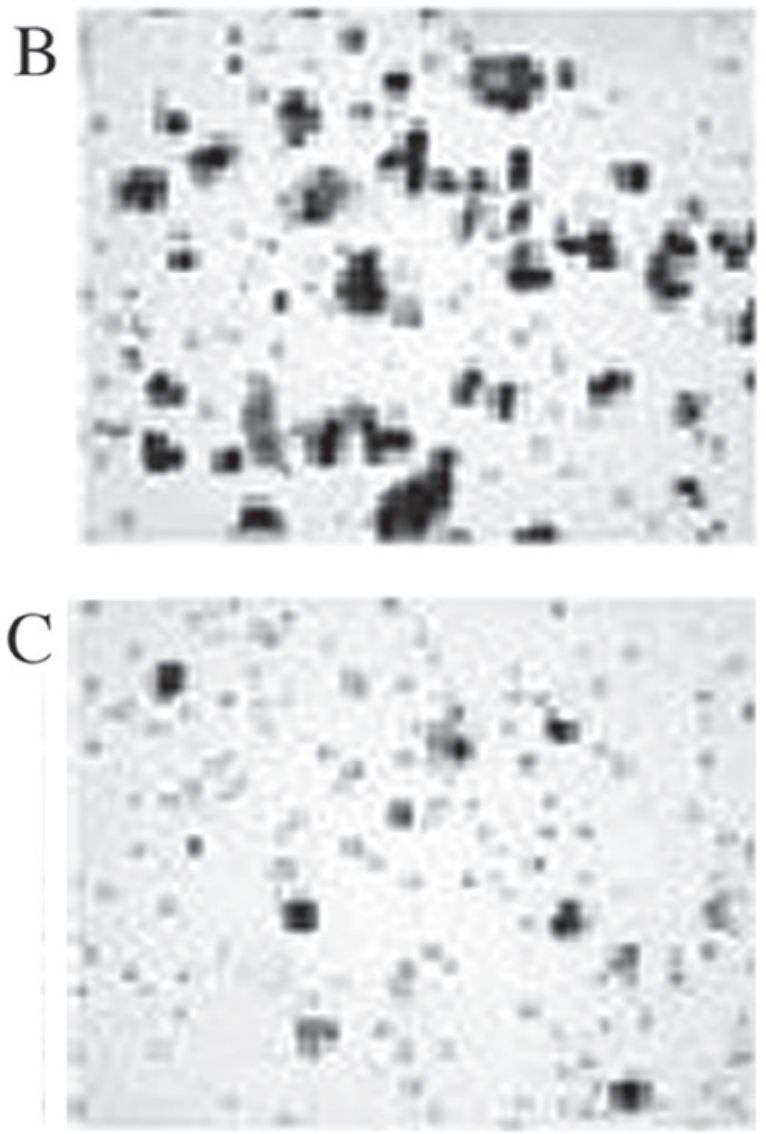

Figure 4. Representative photos showing the migration of MKN45 human gastric cancer cells upon treatment with (A) phosphate-buffered saline (PBS), (B) angiotensin II (Ang II) and (C) the Ang II antagonist losartan.

The AT1 receptor was previously found to be expressed in gastric cancer cell lines and tissues (20). Upregulation of gastric mucosal RAS components gradually increases with time following $H$. pylori infection and appears to associate with the severity of inflammatory cell infiltration (25). The gastric mucosal ATIR mRNA level also appeared to associate with the severity of inflammatory cell infiltration into the gastric mucosa, which reached maximal levels at 12 months after infection in both the antrum and body (19). In the present study, we demonstrated that the expression of Ang II, AT1R, AT2R and the ACE activity were increased in gastric cancer tissue compared to healthy tissues. These results indicate that RAS may be potently involved in the pathogenesis of gastric cancer.
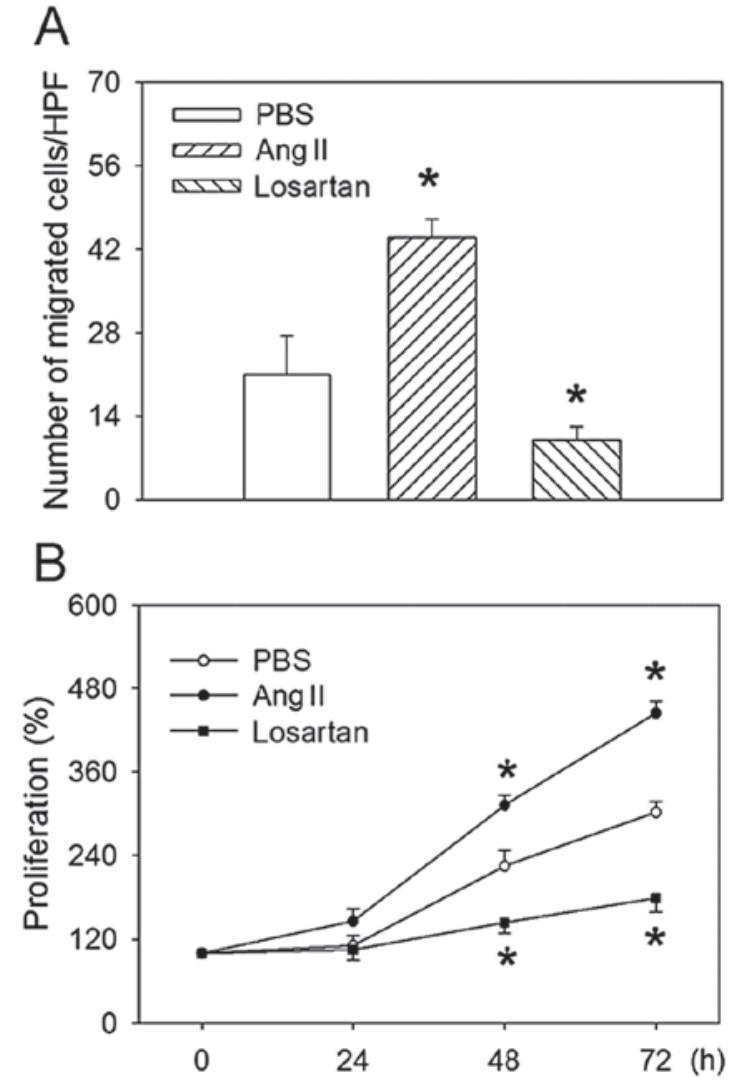

Figure 5. Effects of treatment of MKN45 human gastric cancer cells with phosphate-buffered saline (PBS), angiotensin II (Ang II) and the Ang II antagonist losartan on (A) the number of cells that migrated per high-power field $(\mathrm{HPF})$ and $(\mathrm{B})$ proliferation. Values represent mean $\pm \mathrm{SE}$. ${ }^{*} \mathrm{P}<0.05$ vs. PBS.

It has been shown that insertion/deletion-type polymorphisms in the $A C E$ gene correlate with the development of early gastric cancer (26). ACE inhibitors were shown to inhibit tumor growth through suppression of angiogenesis in a gastric cancer model (23). In the present study, intraperitoneal injection of Ang II into nude mice previously injected with MKN45 cells promoted the size and weight of gastric cancer tumors in gastric cancer mice, whereas the AT1R antagonist losartan significantly inhibited the size and weight of the tumor. These results indicate that Ang II promotes the growth of gastric cancer in immunodeficient mice.

ACE is locally expressed in gastric cancer tissues and insertion/deletion-type polymorphisms in the corresponding gene correlate with metastatic behavior (27). In human gastric cancer cells, Ang II induced the expression of the matrix metalloproteinase-2 (MMP-2) gene (28), which was associated with tumor invasion (29), angiogenesis (30), and metastasis (31). In MKN-28 cells, Ang II induced the expression of MMP-2 and MMP-9 proteins, while its stimulatory effects were efficiently attenuated by the AT1R antagonist (32). Furthermore, ACE was expressed in MKN45 cells (33). In the present study, Ang II significantly promoted, and the AT1R antagonist losartan significantly reduced the migration and proliferation of MKN45 human gastric cancer cells.

In conclusion, the protein levels of Ang II, AT1R and AT2R as well as the activity of the ACE enzyme were increased in gastric cancer tissue from patients. We further propose that 
Ang II promotes the size and weight of gastric cancer tumors in gastric cancer mice and the migration and proliferation of MKN45 human gastric cancer cells. Ang II thus promotes the progression of human gastric cancer. By contrast, the AT1R antagonist losartan significantly reduced the size and weight of tumors in gastric cancer mice and the migration and proliferation of MKN45 human gastric cancer cells. The RAS system may be potently associated with the pathogenesis of gastric carcinogenesis, and therefore, RAS inhibitors may be used for specifically preventing gastric cancer.

\section{References}

1. Jemal A, Bray F, Center MM, Ferlay J, Ward E and Forman D: Global cancer statistics. CA Cancer J Clin 61: 69-90, 2011.

2. Hohenberger P and Gretschel S: Gastric cancer. Lancet 362 $305-315,2003$

3. El-Omar EM, Carrington M, Chow WH, et al: Interleukin-1 polymorphisms associated with increased risk of gastric cancer. Nature 404: 398-402, 2000.

4. El-Omar EM, Rabkin CS, Gammon MD, et al: Increased risk of noncardia gastric cancer associated with proinflammatory cytokine gene polymorphisms. Gastroenterology 124: 1193-1201, 2003.

5. Sugimoto M, Furuta T, Shirai N, et al: Poor metabolizer genotype status of CYP2C19 is a risk factor for developing gastric cancer in Japanese patients with Helicobacter pylori infection. Aliment Pharmacol Ther 22: 1033-1040, 2005.

6. Lu H, Hsu PI, Graham DY and Yamaoka Y: Duodenal ulcer promoting gene of Helicobacter pylori. Gastroenterology 128: 833-848, 2005.

7. Yamaoka Y, Ojo O, Fujimoto S, et al: Helicobacter pylori outer membrane proteins and gastroduodenal disease. Gut 55: 775-781, 2006.

8. Hanahan D and Weinberg RA: The hallmarks of cancer. Cell 100: $57-70,2000$.

9. Yang TS, Yang XH, Wang XD, Wang YL, Zhou B and Song ZS: MiR-214 regulate gastric cancer cell proliferation, migration and invasion by targeting PTEN. Cancer Cell Int 13: 68, 2013.

10. Li P, Chen X, Su L, et al: Epigenetic silencing of miR-338-3p contributes to tumorigenicity in gastric cancer by targeting SSX2IP. PLoS One 8: e66782, 2013.

11. Zhou J, Teng R, Xu C, et al: Overexpression of ER $\alpha$ inhibits proliferation and invasion of MKN28 gastric cancer cells by suppressing $\beta$-catenin. Oncol Rep 30: 1622-1630, 2013.

12. Tian C, Zhang L, Hu D and Ji J: Ghrelin induces gastric cancer cell proliferation, migration, and invasion through GHS-R/NF- $\mathrm{BB}$ signaling pathway. Mol Cell Biochem 382: 163-172, 2013.

13. Lesogor A, Cohn JN, Latini R, et al: Interaction between baseline and early worsening of renal function and efficacy of reninangiotensin-aldosterone system blockade in patients with heart failure: insights from the Val-HeFT study. Eur J Heart Fail 15 1236-1244, 2013.

14. Li P, Sun HJ, Cui BP, Zhou YB and Han Y: Angiotensin-(1-7) in the rostral ventrolateral medulla modulates enhanced cardiac sympathetic afferent reflex and sympathetic activation in renovascular hypertensive rats. Hypertension 61: 820-827, 2013.

15. Suzuki Y, Ruiz-Ortega M, Lorenzo O, Ruperez M, Esteban V and Egido J: Inflammation and angiotensin II. Int J Biochem Cell Biol 35: 881-900, 2003.

16. Le Noble FA, Hekking JW, Van Straaten HW, Slaaf DW and Struyker Boudier HA: Angiotensin II stimulates angiogenesis in the chorio-allantoic membrane of the chick embryo. Eur J Pharmacol 195: 305-306, 1991.
17. Muramatsu M, Yamada M, Takai S and Miyazaki M: Suppression of basic fibroblast growth factor-induced angiogenesis by a specific chymase inhibitor, BCEAB, through the chymase-angiotensin-dependent pathway in hamster sponge granulomas. $\mathrm{Br} \mathrm{J}$ Pharmacol 137: 554-560, 2002.

18. Carl-McGrath S, Ebert MP, Lendeckel U and Röcken C: Expression of the local angiotensin II system in gastric cancer may facilitate lymphatic invasion and nodal spread. Cancer Biol Ther 6: 1218-1226, 2007.

19. Sugimoto M, Ohno T and Yamaoka Y: Expression of angiotensin II type 1 and type 2 receptor mRNAs in the gastric mucosa of Helicobacter pylori-infected Mongolian gerbils. J Gastroenterol 46: 1177-1186, 2011.

20. Kinoshita J, Fushida S, Harada S, et al: Local angiotensin II-generation in human gastric cancer: Correlation with tumor progression through the activation of ERK1/2, NF- $\kappa \mathrm{B}$ and survivin. Int J Oncol 34: 1573-1582, 2009.

21. Ishikawa M, Kitayama J, Yamauchi T, et al: Adiponectin inhibits the growth and peritoneal metastasis of gastric cancer through its specific membrane receptors AdipoR1 and AdipoR2. Cancer Sci 98: 1120-1127, 2007.

22. Savry A, Carre M, Berges R, et al: Bcl-2-enhanced efficacy of microtubule-targeting chemotherapy through Bim overexpression: implications for cancer treatment. Neoplasia 15: 49-60, 2013.

23. Wang L, Cai SR, Zhang $\mathrm{CH}$, et al: Effects of angiotensin-converting enzyme inhibitors and angiotensin II type 1 receptor blockers on lymphangiogenesis of gastric cancer in a nude mouse model. Chin Med J (Engl) 121: 2167-2171, 2008.

24. Huang W, Wu YL, Zhong J, Jiang FX, Tian XL and Yu LF: Angiotensin II type 1 receptor antagonist suppress angiogenesis and growth of gastric cancer xenografts. Dig Dis Sci 53: 1206-1210, 2008.

25. Sugimoto M, Yamaoka Y, Shirai N and Furuta T: Role of renin-angiotensin system in gastric oncogenesis. J Gastroenterol Hepatol 27: 442-451, 2012.

26. Ebert MP, Lendeckel U, Westphal S, et al: The angiotensin I-converting enzyme gene insertion/deletion polymorphism is linked to early gastric cancer. Cancer Epidemiol Biomarkers Prev 14: 2987-2989, 2005

27. Röcken C, Lendeckel U, Dierkes J, et al: The number of lymph node metastases in gastric cancer correlates with the angiotensin I-converting enzyme gene insertion/deletion polymorphism. Clin Cancer Res 11: 2526-2530, 2005.

28. Huang W, Yu LF, Zhong J, et al: Stat3 is involved in angiotensin II-induced expression of MMP2 in gastric cancer cells. Dig Dis Sci 54: 2056-2062, 2009.

29. Tong JJ, Yan Z, Jian R, Tao H, Hui OT and Jian C: RhoA regulates invasion of glioma cells via the c-Jun $\mathrm{NH}_{2}$-terminal kinase pathway under hypoxia. Oncol Lett 4: 495-500, 2012.

30. Murray NP, Reyes E, Badinez L, et al: Effect of androgen blockade on HER-2 and matrix metalloproteinase-2 expression on bone marrow micrometastasis and stromal cells in men with prostate cancer. Scientific World Journal 2013: 281291, 2013. doi:10.1155/2013/281291.

31. Huang T, Chen MH, Wu MY and Wu XY: Correlation between expression of extracellular matrix metalloproteinase inducer and matrix metalloproteinase- 2 and cervical lymph node metastasis of nasopharyngeal carcinoma. Ann Otol Rhinol Laryngol 122: 210-215, 2013.

32. Huang W, Yu LF, Zhong J, et al: Angiotensin II type 1 receptor expression in human gastric cancer and induces MMP2 and MMP9 expression in MKN-28 cells. Dig Dis Sci 53: 163-168, 2008.

33. Carl-McGrath S, Gräntzdörffer I, Lendeckel U, Ebert MP and Röcken C: Angiotensin II-generating enzymes, angiotensinconverting enzyme (ACE) and mast cell chymase (CMA1), in gastric inflammation may be regulated by $H$. pylori and associated cytokines. Pathology 41: 419-427, 2009. 